\title{
Thoracic wall reconstruction using ultrasound images to model/bend the thoracic prosthesis for correction of pectus excavatum
}

\author{
João Gomes Fonseca ${ }^{1,2}$, António H.J. Moreira ${ }^{1,2}$, Pedro L. Rodrigues ${ }^{1,2}$, Jaime C. Fonseca ${ }^{3}$, A.C.M. \\ Pinho $^{4}$, Jorge Correia-Pinto ${ }^{1,2}$, Nuno F. Rodrigues ${ }^{5,6}$, João L. Vilaça ${ }^{1,2,5}$ \\ ${ }^{1}$ Life and Health Sciences Research Institute, SHS, University of Minho, Portugal \\ ${ }^{2}$ ICVS/3B's - PT Government Associate Laboratory, Braga/Guimarães, Portugal \\ ${ }^{3}$ Industrial Electronics Department, University of Minho, Guimarães, Portugal \\ ${ }^{4}$ Mechanical Department, University of Minho, 4800-058 Guimarães, Portugal; \\ ${ }^{5}$ DIGARC - Polytechnic Institute of Cávado and Ave, Barcelos, Portugal \\ ${ }^{6} \mathrm{HASLab} /$ INESC TEC - University of Minho, Braga, Portugal
}

\begin{abstract}
Pectus excavatum is the most common congenital deformity of the anterior thoracic wall. The surgical correction of such deformity, using Nuss procedure, consists in the placement of a personalized convex prosthesis into sub-sternal position to correct the deformity. The aim of this work is the CT-scan substitution by ultrasound imaging for the pre-operative diagnosis and pre-modeling of the prosthesis, in order to avoid patient radiation exposure. To accomplish this, ultrasound images are acquired along an axial plane, followed by a rigid registration method to obtain the spatial transformation between subsequent images. These images are overlapped to reconstruct an axial plane equivalent to a CT-slice. A phantom was used to conduct preliminary experiments and the achieved results were compared with the corresponding CT-data, showing that the proposed methodology can be capable to create a valid approximation of the anterior thoracic wall, which can be used to model/bend the prosthesis.
\end{abstract}

Keywords: CT-scan, pectus excavatum, personalization, reconstruction, ultrasound

\section{INTRODUCTION}

Pectus excavatum is the most common congenital deformity of the anterior thoracic wall, consisting on the abnormal growth of the sternum and ribs [1-2]. Nowadays, surgical correction is carried out in children and young adolescents through Nuss procedure (minimally invasive surgery) [3-5]. This technique involves a prosthesis placement (metallic bar) in sub-sternal position, through two lateral incisions. In most cases, the prosthesis is bended per-operatively, using manual tools, however, Vilaça et al. developed a system which automatically models/bends the surgical prosthesis based on imagiological information (ribs position and maximum latero-lateral distance) - using Computed Tomography (CT) data [6]. Such system ensures a smooth curvature of the surgical prosthesis, a uniform strength distribution over the ribs, the creation of a virtual curvature in asymmetric patients, and predicts precisely the correct size of the prosthesis. The approach reduces surgery time, providing less discomfort and adaptation time to the patient.

Currently, thoracic CT-scan is a compulsory exam to characterize the severity of pectus excavatum, but its necessity is questioned by some specialists of the medical community. Such opinions are supported by the following arguments:

- CT-scan submits the patients to unhealthy radiation, which may lead to long-term serious health problems [7,8];

- Characterization of pectus excavatum severity is not decisive to screening patients for surgery (using Haller index, measured from CT-data), because in a high percentage of cases the surgical correction of pectus excavatum is a cosmetic surgery. So, this surgery essentially depends on patient desire to improve his cosmetic aspect. The main reason to decide is particularly conditioned by the psychological consequences of such deformity in patient life, as social stress, inferiority and insecurity.

Medical Imaging 2012: Ultrasonic Imaging, Tomography, and Therapy, edited by Johan G. Bosch,

Marvin M. Doyley, Proc. of SPIE Vol. 8320, 83200L • (c) 2012 SPIE

CCC code: $1605-7422 / 12 / \$ 18 \cdot$ doi: $10.1117 / 12.911626$

Proc. of SPIE Vol. $832083200 \mathrm{~L}-1$ 
However, CT-data is indispensable for the automatic prosthesis modeling, bringing this approach, presented in [6], the benefits mentioned above. Thus, it is significant, in a short period, to use imaging techniques without radiation to model the surgical prosthesis.

Ultrasound (US) imaging is an interesting alternative to CT-scan, mainly because of economic reasons and it does not use ionizing radiation. However, because of its physical acquisition properties, the pixel intensity and imaging feature (for different structures) are different between these two imaging modalities. The image quality of US systems (very affected by speckle noise) is worse than CT and its field of view is also more limited. Other problems can hinder the capacity of US to have the equivalent information as CT; however, the achieved resolution with US system can be enough to model the prosthesis.

This work aims at the use of 2D US images to reconstruct a thoracic wall approximation - similar to CT-slice - for the posterior, bending of the thoracic prosthesis for pectus excavatum correction. One technique to reconstruct US images is known as freehand US, where the probe is moved by hand, and the resulting sequence of 2D B-scans is registered by either intrinsic (image-based) or extrinsic (position sensing) techniques. In this work, one has chosen to use intrinsic information to reconstruct an approximation of the anterior thoracic wall. Thus, the images are reconstructed in a 2D plane, considering the elevational position of the images constant, since one intends to reproduce the information present in an axial plane of the thoracic wall. The registration process is essentially made between $2 \mathrm{D} / 2 \mathrm{D}$ images and based on in-plane motion. The use of speckle noise reduction to improve the registration process is also explored. The reconstruction, based on several B-scan images, is obtained using the transformations from registration. To validate the proposed methodology, an ultrasound phantom which simulates a thoracic wall partially was employed.

This paper is organized as follows. Section 2 presents the methodology used to make the US reconstruction to obtain the thoracic wall approximation. Section 3 presents and discusses the results of our methodology for different acquisitions. Section 4 presents the concluding remarks.

\section{METHODS}

In this section a methodology is presented to obtain an approximation of the axial (also known as horizontal or transverse) information of the thoracic wall similar to a CT slice, using US imaging. The developed methodology is presented in the following steps:

1) Acquisition of an images sequence along the transverse plane which included the point just before the greatest depression point of the sternum - were the prosthesis will be placed.

2) Selection of a region of interest on the images to ensure an increase in the correlation between two consecutive images.

3) Application of speckle noise reduction (based on Coherence-enhancing Anisotropic Diffusion filtering) to decrease the speckle noise, decrease the influence of the out-of-plane motion decorrelation and enhance the structure features on image. With this step it is expected to improve the following step.

4) Application of rigid image registration (based on block-matching algorithm) to obtain transformations between consecutives images.

5) Reconstruction of the approximation of a thoracic wall slice based on computation of the rigid registration transformations and US images.

\subsection{Image Acquisition}

The image acquisition was initiated in the mid-axillary line and followed the axial plane of the body, ending at the other mid-axillary line (the elevational variation of the probe was considered do not change between images). The $2 \mathrm{D}$ probe was positioned and moved to be in the same axial plane during the scan (Figure 1). Such allowed scanning the anterior thoracic wall and it is expected to get ribs position and the maximum latero-lateral distance. This information shall be necessary for modeling the prosthesis according to [6]. 

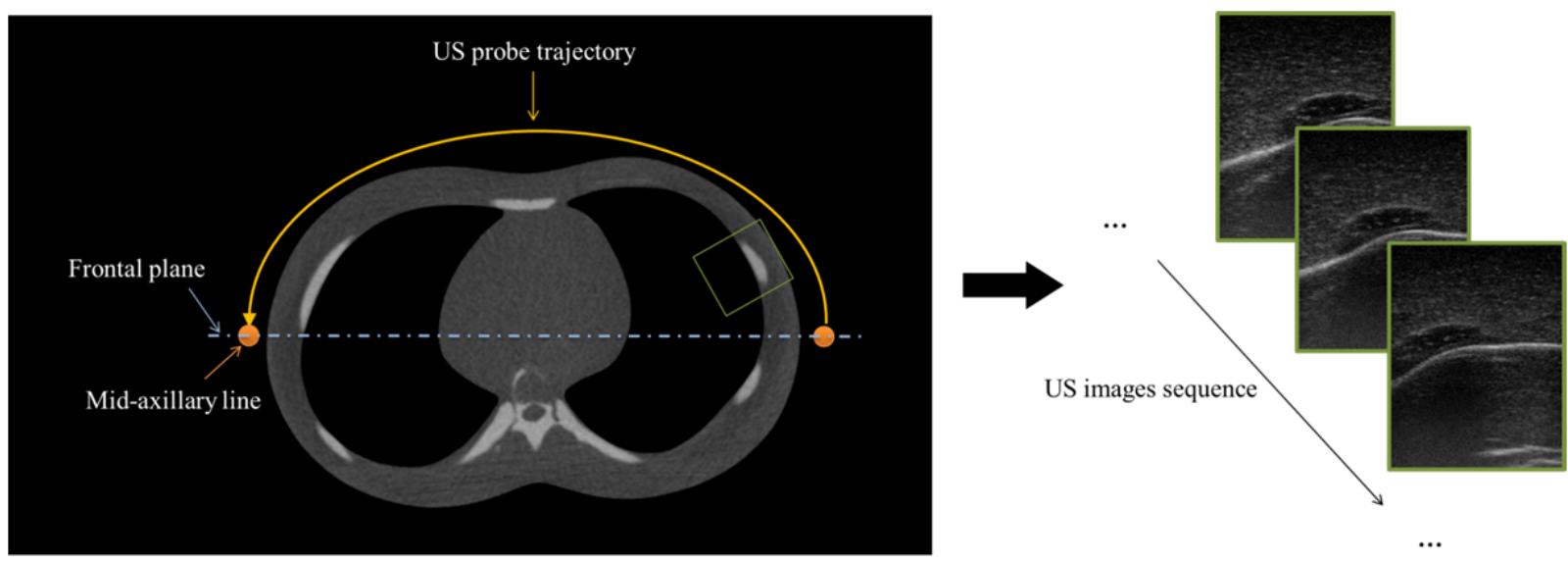

Figure 1. Image of a transverse, horizontal or axial plane of a CT-scan which is used to describe the image acquisition step to obtain a sequence of B-scan images. The blue line represents a frontal plane intersecting the mid-axillary lines (view from the top, orange dots), which are the beginning and ending zones of the image acquisition. The yellow line represents the trajectory of the probe and the green square indicates the field of view of the US probe. In the end, an US images sequence is acquired.

\subsection{Selection of a region of interest}

Initial studies (based on registration errors) revealed that to increase the correlation between US images, it must be eliminated $25 \%$ of the bottom of the acquired images for our procedure and our US system. This coincides with [9], which presents that the decorrelation between consecutive US images are higher in the top and in the bottom of the image, being much higher in the bottom.

\subsection{Speckle noise reduction}

Speckle is known as the grainy appearance that occurs on US images. It is a characteristic that arises from the coherent accumulation of random scatterers (structures smaller than the wavelength of the US wave), so this image artifact is produced by the constructive and destructive interference of such scattered signals $[10,11]$. Such scatterers cause differences in gray levels which do not correspond to the real structure and decrease intensity at boundaries between media of different acoustic impedance, reducing the image quality [12].

The acquired images were pre-processed with a filter for speckle noise reduction. The main purpose of this step was to increase the accuracy of the registration. The used filter was based on Coherence-enhancing Anisotropic Diffusion filtering [13]. It combines concepts of nonlinear diffusion filtering with orientation analysis by means of the structure tensor. Anisotropic diffusion filtering with a diffusion tensor evolves the initial image under an evolution equation:

$$
\frac{\partial I}{\partial t}=\nabla \cdot(D \nabla I)
$$

where $I(x, t)$ is the evolving image, $t$ denotes the diffusion time, and $D$ is the diffusion tensor, a positive definite symmetric matrix that may be adapted to the local image structure. Equation (1) can be solved numerically using finite differences. The expression $A_{i, j}^{k} * I_{i, j}^{k}$ is a discretization of $\nabla .(D \nabla I)$, where $I_{i, j}^{k}$ denotes the approximation of $I(x, t)$ in the pixel $(i, j)$ at the time $k \tau$, where $\tau$ is the time step size, and $A_{i, j}^{k}$ is a mask which varies spatially and temporally when it is convolved with the image.

The coherence filtering was chosen from among others, as adaptive filters (Frost, Lee, Kuan), Speckle Reducing Anisotropic Diffusion (SRAD) and also from different schemes that can be used from it (Implicit Discretization, 
Standard Discretization, Nonnegativity Discretization, Optimized Derivative Kernels and Rotation Invariant). To calculate the best filter, the achieved errors with image registration were used, after applying several controlled transformations. Therefore, the selected filter is a Nonnegativity Discretization scheme from the Coherence-enhancing filtering (Figure 2-B).

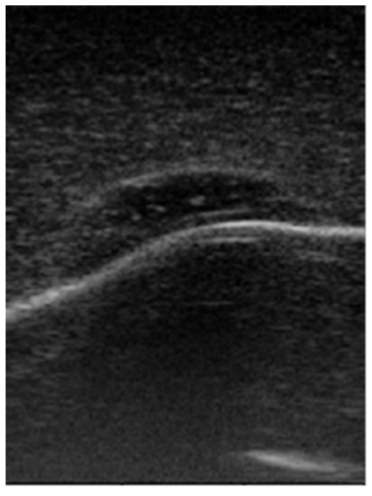

A

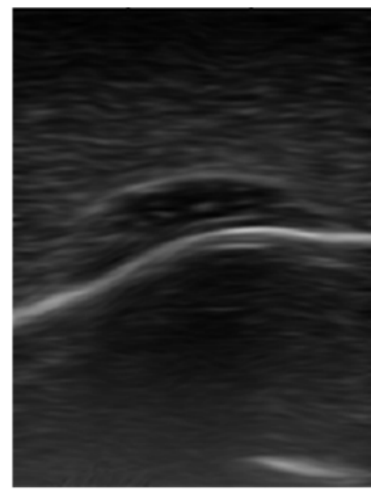

B

Figure 2. A. Original image. B. Filtered image using Nonnegativity Discretization scheme from Coherence-enhancing Anisotropic Diffusion filtering.

\subsection{Image Registration}

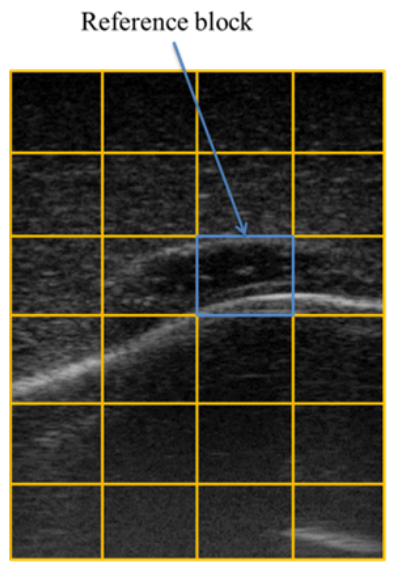

$n+1$

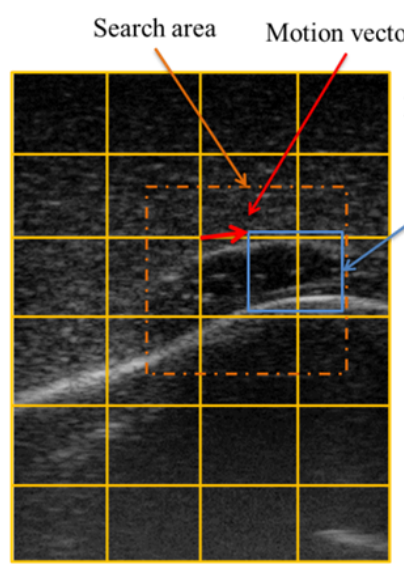

$n$

Rigid registration is performed between two consecutive images, using a block-matching algorithm which provides motion vectors for each block

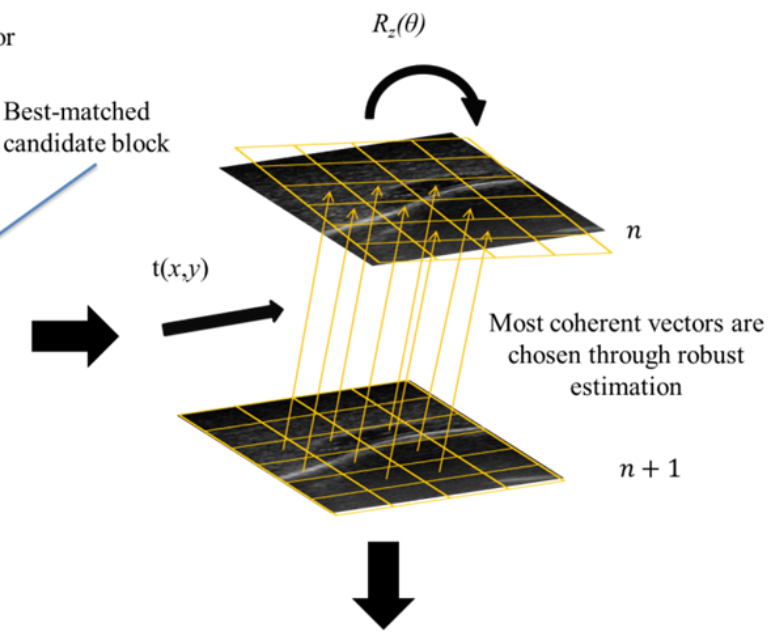

Each operation originates a transformation matrix

$T_{n+1 \rightarrow n}=\left[\begin{array}{cc}R_{z}(\theta) & t(x, y) \\ 0 & 1\end{array}\right]$

Figure 3. Rigid registration method. The block-matching algorithm, which computes the local displacement vectors of the image, is the foundation of registration process. The most coherent vectors are chosen from a multi-scale scheme through robust estimation. In the end, each operation originates a transformation matrix. 
B-scan images were registered using a rigid registration technique, based on block-matching algorithm, presented in [14]. The algorithm assumes that two successive images are not perfectly equivalent from an anatomical point of view. It only assumes local similarities.

These local similarities are computed, through block-matching algorithm, dividing the floating image $I(n+1)$ in several blocks and trying to find in reference image $I(n)$ the best-matched block inside a search area, using a normalized cross-correlation (NCC) as similarity measure (Figure 3). Then, this method tries to find the rigid transformation that matches a maximum of similar regions. So, the transformation is based on the computation of local displacement vectors between two images.

The rigid transformation is then estimated from these matches through a robust regression problem. Instead of being averaged over all the displacements, the transformation is calculated by the majority of matches. It is also important to refer that a multi-scale scheme is used to deal with large displacements and increase the accuracy; this is based on finding the correct displacement vectors using images with different resolutions. It was calculated that only $75 \%$ of the total displacement vectors are used to compute the rigid transformation, using such approach the outliers are eliminated.

So, this image registration method takes as input two images with the same dimensions $(I(n)$ and $I(n+1))$ and as output the transformation $T_{n+1 \rightarrow n}$ which aligns $I(n+1)$ with $I(n)$. The transformation matrix has two in-plane motion components, translation in $x$ and $y t(x, y)$ and rotation around the $z$ axis $R_{z}(\theta)$.

\subsection{Thoracic wall slice reconstruction}

The reconstruction of the thoracic wall, using ultrasound images, is done by applying the transformation matrices to the corresponding images. As shown in figure 4, for each image (except for the first) it will be associated with a transformation. To reconstruct the axial plane from several ultrasound images, the transformations which are above the present transformation must be applied. This allowed the correct localization of a single image (in the space) relatively to previous images, being the first image the main reference. As images are superimposed, a process of transparency was used, being assigned a weight to the gray level of the pixel present on specific image position and to the pixel that is superimposed in that location.

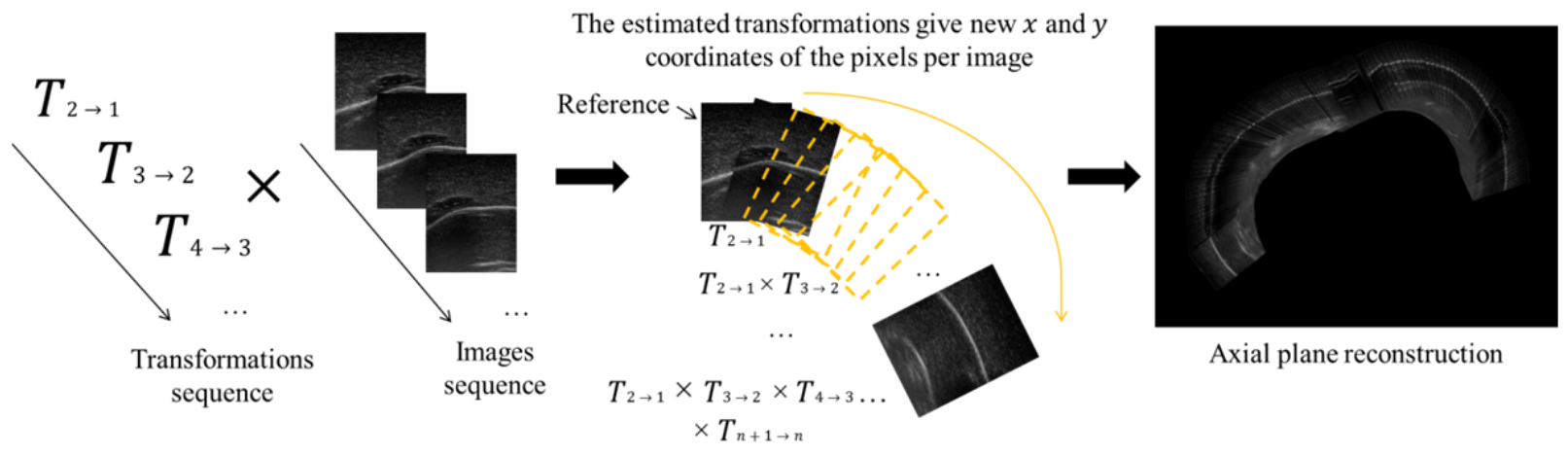

Figure 4. Reconstruction of the axial plane using several B-scan images and transformations associated to each image. To obtain a complete reconstruction, it is necessary to apply each transformation to its own image taking into account the transformations which are before the current image. The first scanned image does not suffer any transformation, it is considered the reference. 


\section{RESULTS AND DISCUSSION}

The used images for the reconstruction of a portion of thoracic wall from ultrasound B-mode images were acquired using the Vivid3 Ultrasound System (GE, Medical Systems).

A $10 \mathrm{MHz}$ linear probe/transducer was used to scan images along an axial plane of the thoracic wall. The scan was made with an acquisition rate of $11 \mathrm{~B}$-scans per second. The images were stored into DICOM format.

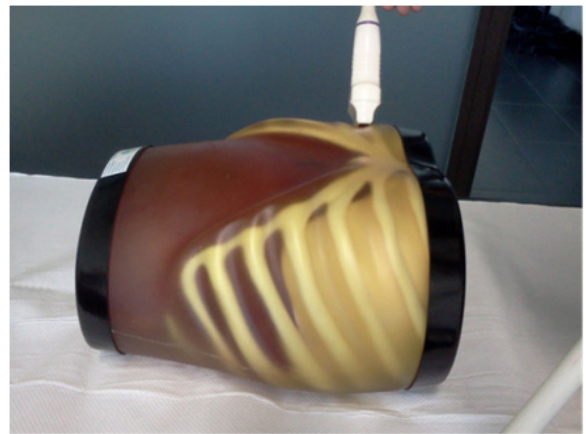

A

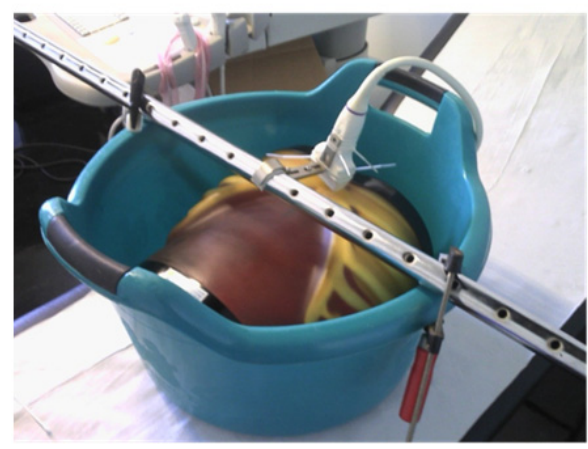

C

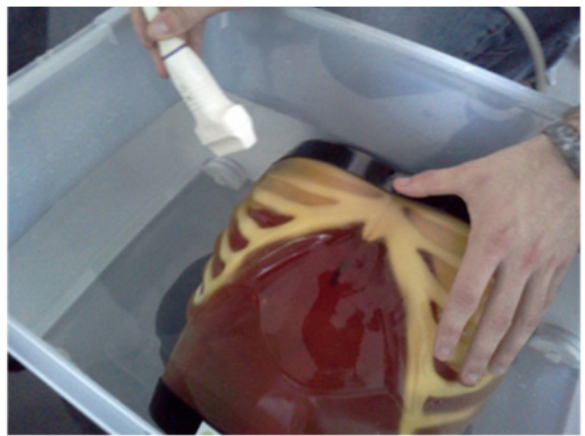

B

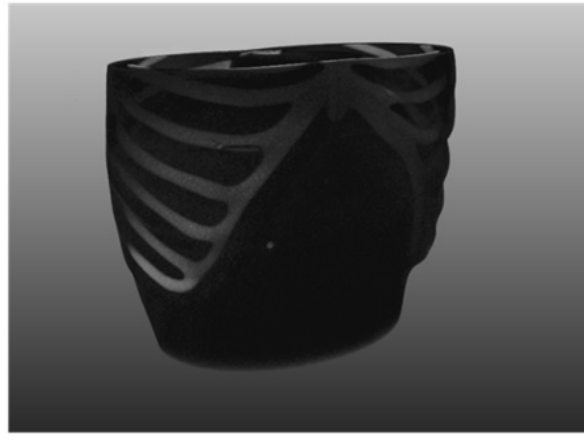

D

Figure 5. Different acquisition approaches. A. Procedure using ultrasonic gel to acquire images from a phantom. B. Procedure using the phantom submerged in water. C. Procedure using the phantom submerged in water with probe supported by metallic support. D. 3D representation of CT-scan of the phantom.

Results were achieved using acquired images from a phantom (Ultrasound Examination Training Model "ABDFAN" Kyoto Kagaku Co., Ltd). The axial plane reconstructions were compared with their corresponding slices from a CT-scan of the phantom (Figure $5-\mathrm{D}$ ). The CT-scan resolution is $512 \times 512 \times 242$ with voxel dimensions of $0.684 \times 0.684 \times 1 \mathrm{~mm}$, consequently, the compared CT-slices were resized according to the image resolution of each US acquisition.

There were used two different approaches to obtain the reconstructions, where different mediums to conduct the acoustic waves between the probe and "skin" were used. One was based on ultrasonic acoustic gel (Figure $5-\mathrm{A}$ ), and the other was based on water, where the phantom was submerged (Figure $5-\mathrm{B}$ and $\mathrm{C}$ ).

To compare the influence of speckle noise reduction in this methodology, in the following sub-sections are presented and compared the reconstructions results with and without speckle filtering. 


\subsection{Procedure using ultrasonic gel}

In this sub-section, the images were acquired using ultrasonic gel as medium, and the scan was performed as it is presented in our methodology. The US image resolution was $0.125 \times 0.125 \mathrm{~mm}$ per pixel in $x$ and $y$ directions with $5 \mathrm{~cm}$ of depth (in $y$ direction).

The results are present in figure 6 with two different reconstructions, one by unfiltered images and the other with speckle noise reduction. The results showed some differences. The position of the bone structures was the main problem, however phantom curvature was well reached, mainly in the filtered image. Some differences between US reconstruction and CT-slice may occur because of differences in CT-slice thickness and the beam thickness (elevational resolution) of the US probe. Due to previous feature, and as the acquisition is done using freehand technique (more instability), the acquired information with US probe tends to reach more volume. Thus, different information from different planes of the CT can be present in the US reconstruction. However, as the prosthesis modulation is extrapolated from some CT-slices to bend the prosthesis (with $13 \mathrm{~mm}$ of thickness); reaching more volume does not influence the automatic system.

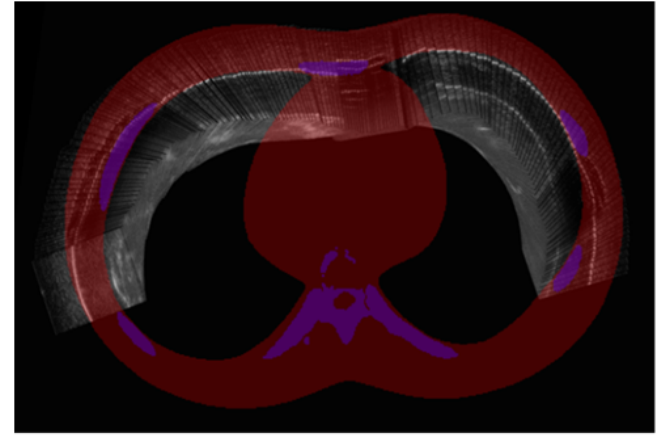

A

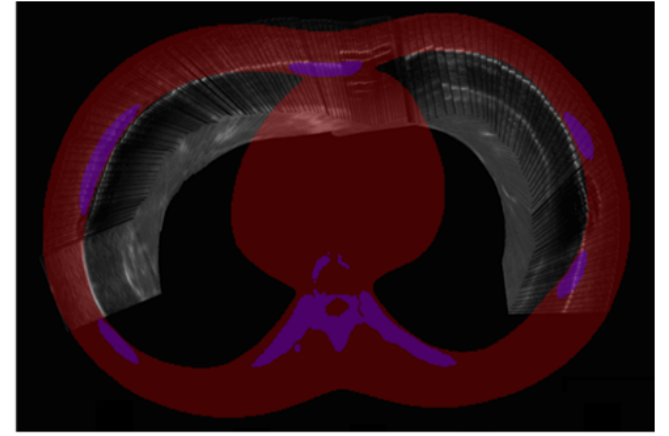

B

Figure 6. Axial plane reconstructions, from procedure using ultrasonic gel, superimposed with CT-data. Violet structures represent the bone structures of the phantom and red structure represents the total constitution of the phantom on a CT-slice. A. Unfiltered images. B. Speckle noise reduction by Coherence-enhancing filtering (Nonnegativity Discretization scheme).

Increasing the frame rate could be also a solution to infer correctly the ribs and the sternum, because the decorrelation between subsequent images would be lesser, as it is presented in [9] and [15]. Nevertheless, our US system presented some limitation in temporal resolution.

Also, other artifacts occurred frequently; the most common is the incorrect contact between surface and probe when the scan is performed. This brought to ultrasound image high black areas and the reverberation artifact in the top of the image, giving less information to the registration process.

So, primary it was tried to use water to eliminate this artifact. Correspondingly, it was possible to evaluate the performance of the methodology when the probe is not in direct contact with the skin, eliminating also the related artifact caused by probe pressure. These results are presented in the following sub-section.

\subsection{Procedure using water (submerged)}

Therefore, in this step the images were acquired by submerging the phantom in water. The ultrasound image resolution was $0.150 \times 0.150 \mathrm{~mm}$ per pixel in $x$ and $y$ directions with $6 \mathrm{~cm}$ of depth, for all acquisitions. This procedure was conducted to understand whether the errors presented in the previous sub-section are related to the direct contact between the probe and the phantom surface. 
The results showed that using water as a medium to conduct the acoustic waves helps the plane reconstruction. Bone structures (ribs and sternum), curvature, internal ("pleura") and external boundaries ("skin") are well defined and positioned using this approach. However, as images present in figure 7 were acquired without any support or contact (just moving and following the external surface away), and the induced instability decreased the registration process. The probe instability can induce to higher transformations and decorrelations that the registration algorithm cannot compute.

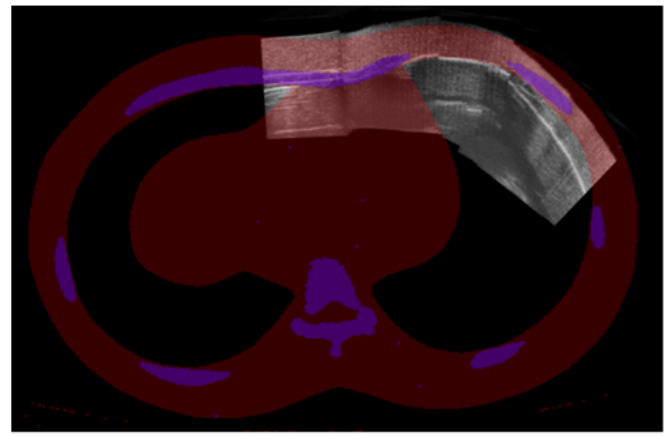

A

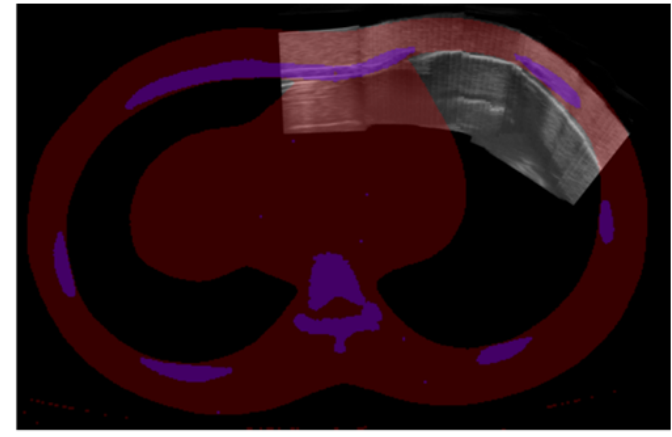

B

Figure 7. Axial plane reconstructions, from submerged phantom, superimposed with CT-data. Violet structures represent the bone structures of the phantom and red structure represents the total constitution of the phantom on a CT-slice. A. Unfiltered images. B. Speckle noise reduction by Coherence-enhancing filtering (Non-negativity Discretization scheme).

Thus, to reduce the instability, the influence of acquiring images with a mechanical support was studied and the results are presented in figure 8. Using a support to acquire images, it was improved the image reconstruction and when they are compared with their correspondent CT-slices, the structures present on image are very similar, both in size and shape. Small structure differences present on image can be related to the propagation time of the sound wave in different mediums and also due to other reasons presented previously. It is important to mention that this acquisition is done by following the surface in rectilinear way (figure $5-\mathrm{C}$ ) and it is observable that bone structures, which appear deeper on ultrasound image, are not visible. So, it is important to explore this solution, drawing and building a support that helps the probe to follow the chest curvature and at the same time give the stability to improve the registration step presented in methodology. Capture the chest curvature, with the probe in the perpendicular position relatively to "skin", is very important because the US waves are attenuated in bone structures and the shadow produced above the rib can be used as a feature to enhance them [16-20], and such only occurs if US beam is perpendicular to bone surface.

Lastly, it is also important to mention that the registration was improved using the image filtering method used in this work, being more evident in figures 6 and 7 than in figure 8. However, it can be notice that having more stability in acquisition augment the correlation between images and errors are lesser, as it is expected when are used speckletracking methods, as block-matching.

\section{CONCLUSION}

This work presents a methodology to reconstruct an approximation of the axial plane of the thoracic wall through several B-scan ultrasound images. The main purpose of such methodology is to replace the CT-scan and its associated radiation from the pectus excavatum correction. Nowadays CT-scan allows the prosthesis personalization for each patient using the system developed by Vilaça et al. Using US imaging, it is possible to avoid the associated problem of radiation and acquire the necessary anatomical information of the thoracic wall. The spatial information of the ribs will be used in the automatic model/bend of the thoracic prosthesis. However, it is necessary to get equivalent accuracy given by the CTscan. 


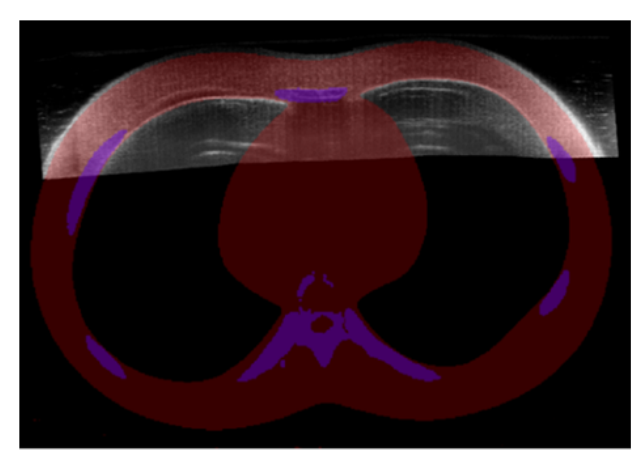

$\mathbf{A}$

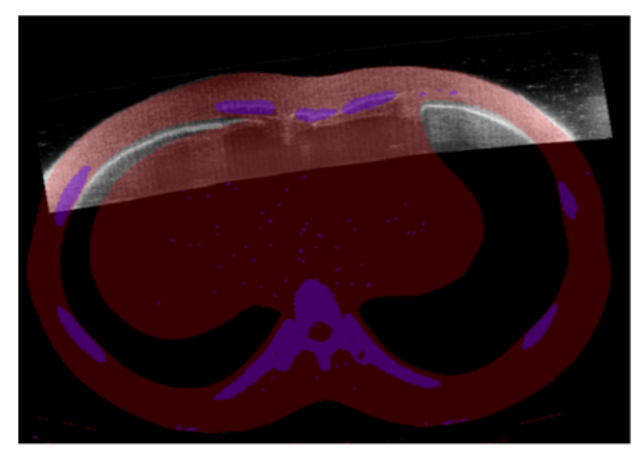

C

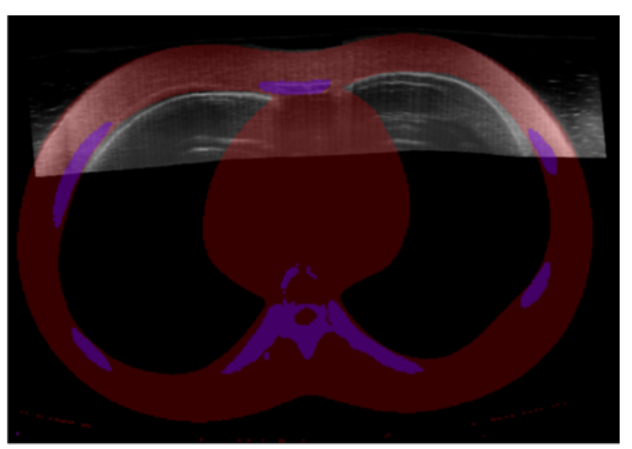

B

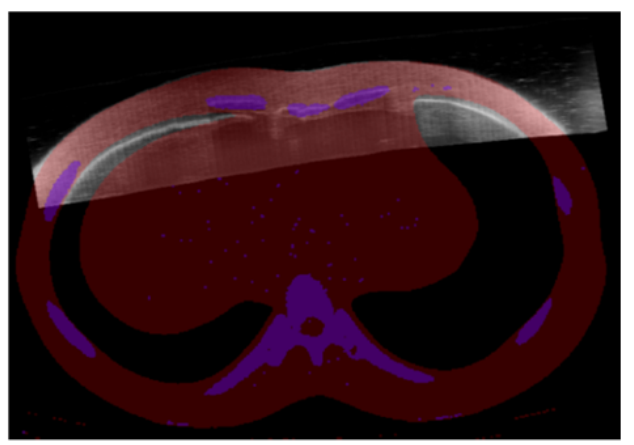

D

Figure 8. Axial plane reconstructions, from submerged phantom using a mechanical support for two different planes, superimposed with CT-data. A. Unfiltered images of the first plane. B. Speckle noise reduction by Coherenceenhancing filtering (Non-negativity Discretization scheme) of the first plane. C. Unfiltered images of the second plane. D. Speckle noise reduction by Coherence-enhancing filtering (Non-negativity Discretization scheme) of the second plane.

Preliminary results from a phantom reveal that present methodology can give an approximation of the external thoracic wall information. The best results were obtained with images submerged in water. The results also revealed that reconstructions using the proposed filtering method present better matching between US reconstructions and CT. However, when the support was used to acquire images, the differences are not so evident. This means that acquisition approach is important to improve image reconstruction, because having more stability in acquisition the correlation between images is improved. Nevertheless, it is relevant to mention that such solution could be not valid to use in clinical cases, because it could be difficult to create conditions to submerge human bodies in water and also have supporters to assist the image acquisition. However, our solution can be less expensive and easy to implement than sensing freehand techniques (with a position sensor), yet it can infer in-plane motion and out-of-plane motion [21]. So, to demonstrate the viability of our solution, it is necessary to develop a mechanical system which brings more stability to the acquired images and follows the curvature of the thoracic wall. To improve and to turn it more robust, it should be introduced in the image reconstruction more complex algorithms based on image features as: speckle decorrelation, estimation of the out-of-plane motion, non-rigid registration, etc. [9, 15, 22-25].

Once when the reconstruction system is validated, reconstructions should be performed in vivo with patients with pectus excavatum. Bone segmentation has to be associated to this methodology to enhance the presence of ribs and to be possible to model the prosthesis using their surface points. To compare the accuracy of ultrasound reconstruction and $\mathrm{CT}$, comparisons should be performed between modeled prosthesis by these two imaging modalities. 


\section{ACKNOWLEDGEMENTS}

The authors acknowledge to Foundation for Science and Technology (FCT) - Portugal for the fellowships with the references: SFRH/BPD/66151/2009; and, SFRH/BPD/46851/2008. This work was also supported by FCT R\&D project PTDC/SAU-BEB/103368/2008.

\section{REFERENCES}

[1] Aloi, I., Braguglia, A. and Inserra, A., "Pectus excavatum," Paediatrics and Child Health 19, 132-142 (2009).

[2] Fokin, A. A., Steuerwald, N. M., Ahrens, W. A., and Allen, K. E., "Anatomical, Histologic, and Genetic Characteristics of Congenital Chest Wall Deformities," Thoracic and Cardiovascular Surgery 21, $44-57$ (2009).

[3] Mao, Y.-Z., Tang, S.-T., Wang, Y., Tong, Q.-S., and Ruan, Q.-L., "Nuss operation for pectus excavatum: a single-institution experience," World Journal of Pediatrics 5, 292-295 (2009).

[4] Matos, A. C., Bernardo, J. E., Fernandes, L. E., and Antunes, M. J., "Surgery of chest wall deformities," European Journal of Cardio-thoracic Surgery 12, 345-350 (1997).

[5] Iida, H., "Surgical repair of pectus excavatum," Gen Thorac Cardiovasc Surg 58(2), 55-61 (2010).

[6] Vilaça, J. L., Pinho, A., Correia-Pinto, J., Fonseca, J., Peixinho, N., "System for automatic and personalized modelling/bending of surgical prosthesis for correction of pectus excavatum based on pre-surgical imagiology information," WO2009/035358 (2009).

[7] Frush, D. P., Donnelly, L. F. and Rosen, N. S., "Computed Tomography and Radiation Risks: What Pediatric Health Care Providers Should Know," Journal of the American Academy of Pediatrics 112, 951-957 (2003)

[8] Brenner, D. J. and Hall, E. J., "Computed Tomography - An incressing source of radiation exposure," The new england journal of medicine 357, 2277-2284 (2007)

[9] Hassenpflug, P., Prager, R. W., Treece, G. M. and Gee, A. H., "Speckle classification for sensorless freehand 3D ultrasound," Ultrasound in Medicine and Biology 31(11), 1499-1508 (2005)

[10] Jan D'hooge, [Principles and different techniques for speckle tracking, in Myocardial Imaging: Tissue Doppler and Speckle Tracking ], Blackwell Publishing, 17-25 (2007).

[11]Dantas, R. G., Costa, E. T., Leeman, S., "Ultrasound speckle and equivalent scatterers," Ultrasonics 43, 405-420 (2005).

[12] Ali, M., Magee, D., Dasgupta, U., "Signal Processing Overview of Ultrasound Systems for Medical Imaging," Texas Instruments, (2008).

[13] Weickerty, J. and Scharr, H., "A Scheme for Coherence-Enhancing Diffusion Filtering with Optimized Rotation Invariance".

[14] Ourselin, S., Roche, A., Subsol, G., Pennec, X., and Ayache, N., "Reconstructing a 3D structure from serial histological sections," Image and Vision Computing 19, 25-31 (2000)

[15] Gee, A. H., Housden, R. J., Hassenpflug, P., Treece, G. M., Prager, R. W., "Sensorless freehand 3D ultrasound in real tissue: Speckle decorrelation without fully developed speckle," Medical Image Analysis 10, 137-149 (2006)

[16] Huisman, H. and Karssemeijer, N., "Chestwall Segmentation in 3D Breast Ultrasound Using a Deformable Volume Model”, Springer-Verlag Berlin Heidelberg 2007, 245-256 (2007).

[17] Foroughi, P., et al., "Ultrasound Bone Segmentation Using Dynamic Programming", IEEE Ultrasonics Symposium, 2523-2526 (2007).

[18] Chang, R.-F., et al., "Rib Detection for Whole Breast Ultrasound Image", SPIE Medical Imaging 2008: Computer-Aided Diagnosis, Proc. SPIE 6915 (2008).

[19] Doctor, A., Vondenbusch, B., and Kozak, J., "Bone segmentation applying rigid bone position and triple shadow check method based on RF data," Acta of Bioengineering and Biomechanics 13, 3-11 (2011).

[20] Kumar, A. J. and Taylor, R. H., "Understanding Bone responses in B-mode Ultrasound Images and Automatic Bone Surface extraction using a Baysian Probabilistic Framework", SPIE Medical Imaging 2004: Ultrasonic Imaging and Signal Processing, Proc. SPIE 5373 (2004).

[21] Treece, G. M., Prager, R. W., Gee, A. H. and Berman, L., "Correction of probe pressure artifacts in freehand 3D ultrasound," Medical Image Analysis 6, 199-214 (2002) 
[22] Prager, R. W., Gee, A. H., Treece, G. M., Cash, C. J. C. and Berman, L. H., "Sensorless Freehand 3-D Ultrasound using regression of the echo intensity," Ultrasound in Medicine and Biology 29 (3), 437-446 (2003)

[23] Housden, R. J., Gee, A. H., Treece, G. M. and Prager, R. W., "Subsample interpolation strategies for sensorless freehand 3D ultrasound," Ultrasound in Medicine and Biology 32(12), 1897-1904 (2006)

[24] Housden, R. J., Gee, A. H., Treece, G. M. and Prager, R. W., "Sensorless reconstruction of unconstrained freehand 3D ultrasound data," Ultrasound in Medicine and Biology 33(3), 408-419 (2007)

[25] Lang, A., Mousavi, P., Gill, S., Fichtinger, G. and Abolmaesumi, P., "Multi-modal registration of speckletracked freehand 3D ultrasound to CT in the lumbar spine," Medical Image Analysis, (2011) 